Athens Journal of Health \& Medical Sciences - Volume 7, Issue 3, September 2020 - Pages 157-170

\title{
mRNA Expressions of Specific Gamma-Glutamyl Transferases in Molecular Subtypes of Breast Cancer
}

\author{
By Dilek Gelen-Güngör*, Ece Miser-Salihoglü, Semra Demokan ${ }^{*}$, \\ Karanlik Hasan ${ }^{+} \&$ Sevgi Yardim-Akaydin
}

\begin{abstract}
An increased risk of breast cancer has been reported in individuals with elevated levels of gamma-glutamyl transferase (GGT). GGT1 was the only enzyme used for diagnosis in clinic and human genome contains additional related genes or sequences besides GGT1. From the perspective of amino acid sequences, genes showing substantial similarity (GGT5, GGT6, and GGT7) to GGT1 have been identified. The aim of this study was to investigate the mRNA expressions of GGT1, GGT5, GGT6, and GGT7 in 58 breast cancer patients' tissue samples by qRT-PCR method. In total, mRNA expressions of GGT5 and GGT7 increased and GGT6 decreased in tumor tissues than those in normal tissues of the same patients ( $p<0.145, p<0.003$ and $p<0.05$, respectively). Among molecular subtypes, GGT7 expressions were significantly higher in tumor tissues than those in normal tissues of the patients in Luminal A group $(p<0.009)$. Over-expression of GGT7 was observed in almost half of the patients. The research showed mRNA expressions of GGT1, GGT5, GGT6, and GGT7 in breast cancer. Among the four genes, we obtained surprising results for GGT7 and we believe that the activity of this gene should be examined in breast cancer.
\end{abstract}

Keywords: GGT1, GGT5, GGT6, GGT7, breast cancer, mRNA expressions

\section{Introduction}

Among the most common cancers in women, breast cancer is seen in one out of every four females. A total of 17,571 Turkish women with breast cancer, which is the first-line cancer type in women all around the world, was diagnosed in 2013. Breast cancer incidence in Turkish women was observed as 45\% between 50-69 years of age and $40 \%$ between 25-49 years of age (Türkyilmaz et al. 2018).

Breast carcinomas are highly heterogeneous tumors with clinical signs/ symptoms/treatment responses as well as biological behaviors. According to recent research, immunophenotypic and molecular classification have been shown to be much more prognostic and predictive than classification based on basic clinicopathological parameters such as morphology, tumor histological subtype, and histologic grade applied for many years (Banerji et al. 2012, Carey et al. 2006, Rouzier et al. 2005, Tran and Bedard 2011).

\footnotetext{
*Research Assistant, Gazi University, Turkey.

${ }^{ \pm}$Research Assistant, Gazi University, Turkey.

*Professor, Istanbul University, Turkey.

${ }^{+}$Professor, Istanbul University, Turkey.

•Professor, Gazi University, Turkey.
} 
The first molecular classification of breast carcinomas was performed by Perou et al. by using DNA microarray (microarray) method in 2000 (Perou et al. 2000). According to this classification, breast carcinomas are divided into 4 subtypes; i. Luminal, ii. HER2 (+), iii. Basal-like, and iv. Normal breast-like. In the light of advanced molecular knowledge up to date, breast carcinomas are divided into 5 subgroups (Luminal A, Luminal B, HER (+), Luminal B-HER (+), triple negative) based on the levels of hormones (estrogen, ER and progesterone, PR) and HER2 expression. In recent years, the Ki-67 proliferation index has also been added to this classification, especially in the clinical-oncological predictive direction (Carey et al. 2006, Tran and Bedard 2011, Cheang et al. 2009, Dent et al. 2007, Yang et al. 2007)

Gamma-glutamyl transferase (5-L-glutamyl-peptide: amino acid 5-glutamyl transferase; GGT; EC 2.3.2.2) is an enzyme with ecto-activity located on the outer surface of plasma membranes of cells. GGT is a dimeric glycoprotein composed of a heavy and light subunit bound to noncovalent bonds and is processed from a single chain precursor by an autocatalytic cleavage in prokaryotes and eukaryotes (MyBioSource 2006, InterPro 2017, Fornaciari et al. 2014). The enzyme has an auto-cleavage function and this function is linked to the nucleophilic threonine (Thr 381) in the peptide sequence (Brenda 2019). GGT is found in the structure of membranes of almost all cells, mainly in epithelial tissues with secretory or absorptive functions. While the enzyme is expressed by the cells of many organs, the highest GGT activity is found in the kidney, duodenum, small intestine, and bile duct cells. However, plasma GGT is thought to be derived from the liver (Fornaciari et al. 2014). It has critical functions in the glutathione metabolism and the conversion of Leukotriene C4 (LTC4) to Leukotriene D4 (LTD4) (Hanigan 2014). The glutathione synthesized in the cell is transported extracellularly by GGT. GGT can break the glutamyl peptide bond in glutathione and other proteins and transfer glutamyl residues to an acceptance such as amino acid, peptide or water (MyBioSource 2006, InterPro 2017, Hanigan 2014).

An increased risk of breast cancer has been reported in individuals with elevated levels of GGT. In a recent study, serum GGT activity was found to be slightly higher in breast cancer patients compared to the control group (Shackshaft et al. 2017) In addition, positive associations were found between serum GGT activity and development of ER+, ER- and $\mathrm{PR}+$ breast cancers compared to controls and inverse associations between GGT levels and PR- breast cancers compared to PR+. Staudigl et al. did not found any relationship between GGT enzyme activity and hormone receptor and HER2-status (Staudigl 2015). On the other hand, a positive correlation was reported between increased GGT activity and breast cancer incidence in only premenopausal women (Fentiman and Allen 2010) However, increased GGT levels were explained an independent risk-factor for breast cancer by Van Hemelrijck et al. (2011). Despite the accumulation of evidence that increased GGT levels may be a risk factor for breast cancer, it is not known which activity or type of GGT is responsible for this. In collaboration with the HUGO (Human Genome Organization) and Human Genome Nomenclature Committee (HGNC), possible active genes resembling GGT1, the only enzyme used for clinical diagnosis, with nucleotide and amino acid sequences have been 
identified (Heisterkamp et al. 2008). 13 genes of GGT family were detected when GGT associated human gene sequences were searched by using genomic and cDNA databases. A real protein entity could not be shown for other genes except for GGT1, GGT5 (formerly GGL, GGTLA1/GGTrel) and GGT7 (formerly GGTL3, GGT4). Experimentally, it has been shown that only GGT1 and GGT5 turn into a protein with enzymatic activity. GGT6 (formerly rat ggt6 homologue) and GGT7 carries $47 \%$ and 52\% amino acid sequence identity to GGT1 and GGT5, respectively, which are better characterized than other family members (Heisterkamp et al. 2008).

In studies with GGT1 and GGT5 null mutants, GGT1 has been shown to play a role mainly in glutathione metabolism (Carter et al. 1997, 1998) and GGT5 in leukotriene metabolism (Han et al. 2002). In a study on glioblastoma, the demonstration that GGT7 reduction increases the amount of cellular reactive oxygen species suggests that it may be related to GSH metabolism (Bui et al. 2015). The function of GGT6 has not yet been described.

In our previous study with a small group of various types of cancer, we achieved interesting results in GGT1, GGT5 and GGT6 mRNA expressions in breast cancer (Yardım-Akaydın et al. 2017), and our main goal in this study was to examine the expressions of the same GGT genes by including GGT7 in a larger breast cancer patient population. For this purpose, we examined the mRNA expression levels of GGT1, GGT5, GGT6 and GGT7 genes in tumor and matchednormal tissues of patients with breast cancer and the expression differences of these genes among the molecular subtypes of the breast cancer.

\section{Materials and Methods}

Fifty-eight patients, who applied to Istanbul University, Oncology Institute, Clinical Oncology Department, Oncology Surgical Unit and were diagnosed with breast cancer and had operation due to their illness, were included in the study. The patients were informed for participation in the study with approval prior to the operation date and their voluntary approvals were obtained. One $\mathrm{cm}^{3}$ size $(100$ $\mathrm{mg}$ ) tissue samples (tumor and matched-normal tissues) were taken during the operation from patients. Eight healthy women who applied to Istanbul University, Oncology Institute, Clinical Oncology Department, Oncology Surgical Unit for macromastia and for breast reduction surgery and without a history of breast cancer story in family, were included as the control group. The main characteristics and laboratory results of patients were given in Table 1. While the tumor and matched-normal tissues were taken from breast cancer patients in surgery, pathological examination of the tissues were performed simultaneously and excised tissue is stored at $-80^{\circ} \mathrm{C}$ immediately. This study was approved by the Clinical Research Ethics Committee of Istanbul, Faculty of Medicine (2016/419106748). 
Demographic Characteristics and Laboratory Tests of the Patients and Controls

Table 1. The Main Characteristics and Laboratory Results of Patients

\begin{tabular}{|ll|c|}
\hline Parameters & Patients (n=58) & Controls (n=8) \\
\hline Age, mean (SD) & $53.1(12.0)$ & $36.3(9.6)$ \\
Menopause Status & & \\
$\quad$ Premenopausal, n (\%) & $33(56.9)$ & $6(75)$ \\
Postmenopausal, n (\%) & $25(43.1)$ & $2(25)$ \\
Stage, n (\%) & & - \\
$\quad$ I & $5(8.6)$ & - \\
II & $23(39.7)$ & - \\
III & $30(51.7)$ & - \\
Lymph Node Involvement, n (\%) & $5(8.6)$ & - \\
Tumor Location, n (\%) & & - \\
$\quad$ Right side & $31(53.5)$ & - \\
Left side & $26(44.8)$ & - \\
right +left sides & $1(1.7)$ & - \\
\hline Laboratory tests & & - \\
$\quad$ Estrogen Receptor, mean (SD) & $43.5(43.3)$ & - \\
Progesterone Receptor, mean (SD) & $21.5(32.8)$ & \\
Ki67, mean (SD) & $40.8(26.2)$ & \\
\hline
\end{tabular}

Molecular classification of the tumor tissues was performed according to the presence of estrogen/progesterone hormone receptor, Ki67, and c-erbB2 (HER2), which are given below (Table 2).

Table 2. Parameters Used in the Classification of Breast Cancer Patients

\begin{tabular}{|l|l|c|}
\hline Molecular Subtype & Parameter & N \\
\hline Luminal A & ER(+)/PR(+)/Ki67 less-than 25 & $16(27.6)$ \\
\hline Luminal B & ER(+)/PR(+)/Ki67 more-than 25 & $8(13.8)$ \\
\hline Luminal B-Her2 & ER(+)/PR(+)/Ki67 more-than 25, c-erbB2(+3) & $9(15.5)$ \\
\hline HER2 positive & ER(-)/PR(-)/c-erbB2(+3) & $9(15.5)$ \\
\hline Triple negative & ER(-)/PR(-)/c-erbB2(-) & $16(27.6)$ \\
\hline
\end{tabular}

\section{Quantitative Real-Time PCR (qRT-PCR) Analysis}

To obtain RNA, all tumor and matched-normal tissues were homogenized in TRIzol Reagent (Invitrogen, Carlsbad, CA, USA). After RNA isolation, cDNA synthesis reactions were performed with SensiFAST cDNA Synthesis Kit (Bioline USA Inc., USA). Both of two methods were applied according to the manufacturer's instructions. The primer sequences used were as follows:

GGT1: Fw-5'- TGACCTTCAGGAGAACGAGA -3', Rv-5'- TCTTCTTCA

TGGCTCTGCGT -3'

GGT5: Fw-5' -CTCCAAGGTCTGCTCGGAT-3', Rv-5'-

GTTGTCACATTGTAGATGGTG-3' 
GGT6: Fw-5'-ATTCCACGGCCCTGACATCA-3', Rv-5'-

CCATCAGCATGGCAAAGTAGT-3'

GGT7: Fw-5' - ACACCGACCCGGACTCCTT -3', Rv-5'ACGGGTCTTTGCGCGTCTC-3'

Internal reference, $\beta$-actin: Fw-5'-GTCTTCCCCTCCATCGTG-3'

Rv-5'-AGGGTGAGGATGCCTCTCTT-3'

PCR conditions: $95^{\circ} \mathrm{C}$ for $2 \mathrm{~min}, 95^{\circ} \mathrm{C}$ for $5 \mathrm{sec}, 65^{\circ} \mathrm{C}$ for $10 \mathrm{sec}, 72^{\circ} \mathrm{C}$ for 20 sec, for 40 cycles. Measurements of GGTs mRNA expression levels in tumor and matched-normal tissues were carried out in parallel. qRT-PCR was done in a AriaMx Real-Time PCR System (Agilent Technologies, Santa Clara, CA, USA). The experiments were performed in triplicate. Target gene mRNA expressions were quantified and standardized according to the $\beta$-actin reference gene signal. Relative quantification values were calculated by the formula of the Pfaffl method shown below (Pfaffl 2001).

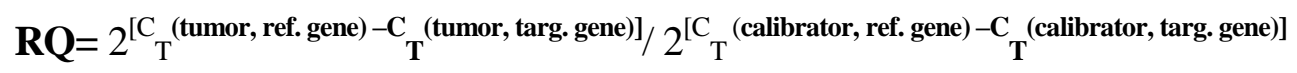

Target mRNA expressions were calculated in tumor and matched-normal tissues (calibrator sample), compared to reference mRNA expressions on the basis of the difference between $\mathrm{CT}$ values of the target and reference genes $(\Delta \mathrm{CT})$ (as proportional).

\section{Statistical Analysis}

All data was expressed as mean (standard deviation, SD). The homogeneity of the data was evaluated with the Kolmogorov-Smirnov test. For non-homogenous (non-normal distribution) data, matched-normal tissue and tumor tissue changes of the each gene in the same patient were analyzed by Wilcoxon signed rank test. $\mathrm{P}$ values of less than 0.05 were regarded as statistically significant. Statistical analyzes were performed using the SPSS 22 Trial Package Program (SPSS Inc, USA).

\section{Results}

mRNA Expression Levels of GGTs

According to relative quantification values of GGTs, tumor mRNA expression greater than 1.5 fold relative to the corresponding gene expression in matchednormal tissues was considered to be an overexpression.

According to the qRT-PCR results, comparison of normal tissue and tumor tissue mRNA expression levels were presented in Table 3. The tumor tissue expression levels of GGT1 was found to be significantly higher than those of matched-normal tissues $(\mathrm{p}=0.02)$ in the total breast group. Among the sub-groups, mean expression levels of GGT1 was higher in matched-normal tissues than those 
in tumor tissues in Luminal B-Her2 (+) group [0.39 (0.46) vs $0.08(0.17)$, respectively, $\mathrm{p}=0.028]$. Although GGT5 mRNA levels were generally higher in tumor tissues, no statistically significant difference was observed between matchednormal and tumor tissue expression levels $(\mathrm{p}=0.145)$. Mean values of GGT6 mRNA expression levels were found to be higher in matched-normal tissues than those in tumor tissues in the total breast group ( $\mathrm{p}=0.045)$. When subgroups were evaluated, tumor tissue expression levels were higher in the Her2 $(+)$ group $(n=9)$, but statistical significance was borderline $(\mathrm{p}=0.069)$. In both of total breast and the Luminal A groups, mean GGT7 expression values were significantly higher in tumors than those in the matched-normal tissues $[0.2132(0.2500)$ vs 0.11349 (0.1192), respectively, $\mathrm{p}=0.003$ for the total group and 0.3690 (0.3543) vs 0.2060 (0.2435), respectively, $\mathrm{p}=0.009$ for Luminal A group]. Similarly, higher tumor tissue expression levels were also observed in Triple-negative $(n=15)$ and Her2positive $(n=9)$ groups, but statistical significance was borderline $(\mathrm{p}=0.069$ and $\mathrm{p}=0.063$, respectively). When the normal tissue mRNA expression of the patient and healthy controls were compared, no statistically significant difference was observed in any of the four GGT genes ( $\mathrm{p}>0.05)$.

GGT1, GGT5 and GGT6 mRNA expressions were not observed in normal tissues of 16, 23 and 16 of 58 patients (respectively), and in tumor tissues of 16, 16 and 16 of 58 patients. All 3 genes were expressed in the tissues of 4 out of 8 healthy controls. Except for only one normal tissue of the patients, all normal and tumor tissues had GGT7 expressions. For all the patients, 25.8\% (15/58) of patients for GGT1, 15.5\% (9/58) of patients for GGT5, 15.5\% (9/58) of patients for GGT6, and 46.6\% (27/58) of patients for GGT7 showed higher mRNA expression fold changes (Threshold for fold change is greater than and equal to 1.5) (Figure1).

According to the subgroups, GGT1 overexpressions were observed as $35.7 \%$ for Luminal A, $50 \%$ for Luminal B, $22.2 \%$ for Luminal B+Her2(+), 33.3\% for Her2(+), and $26.7 \%$ for Triple(-) groups (Figure 2a). GGT5 overexpressions were observed as $57.1 \%$ for Luminal A, $40.0 \%$ for Luminal B, $62.5 \%$ for Luminal $\mathrm{B}+\mathrm{Her} 2(+), 55.6 \%$ for Her2(+), and $38.5 \%$ for Triple(-) groups (Figure 2b). GGT6 overexpressions were observed as $41.6 \%$ for Luminal A, $50.0 \%$ for Luminal B, $28.6 \%$ for Luminal B+Her2(+), 33.3\% for Her2(+), and $62.5 \%$ for Triple(-) groups (Figure 2c). GGT7 overexpressions were observed as $56.3 \%$ for Luminal A, $37.7 \%$ for Luminal B, $28.6 \%$ for Luminal B+Her2(+), $77.8 \%$ for Her2(+), and $56.3 \%$ for Triple(-) groups (Figure 2d). 
Figure 1. Scatter Plot of GGTs' Expressions in All Breast Cancer Patients

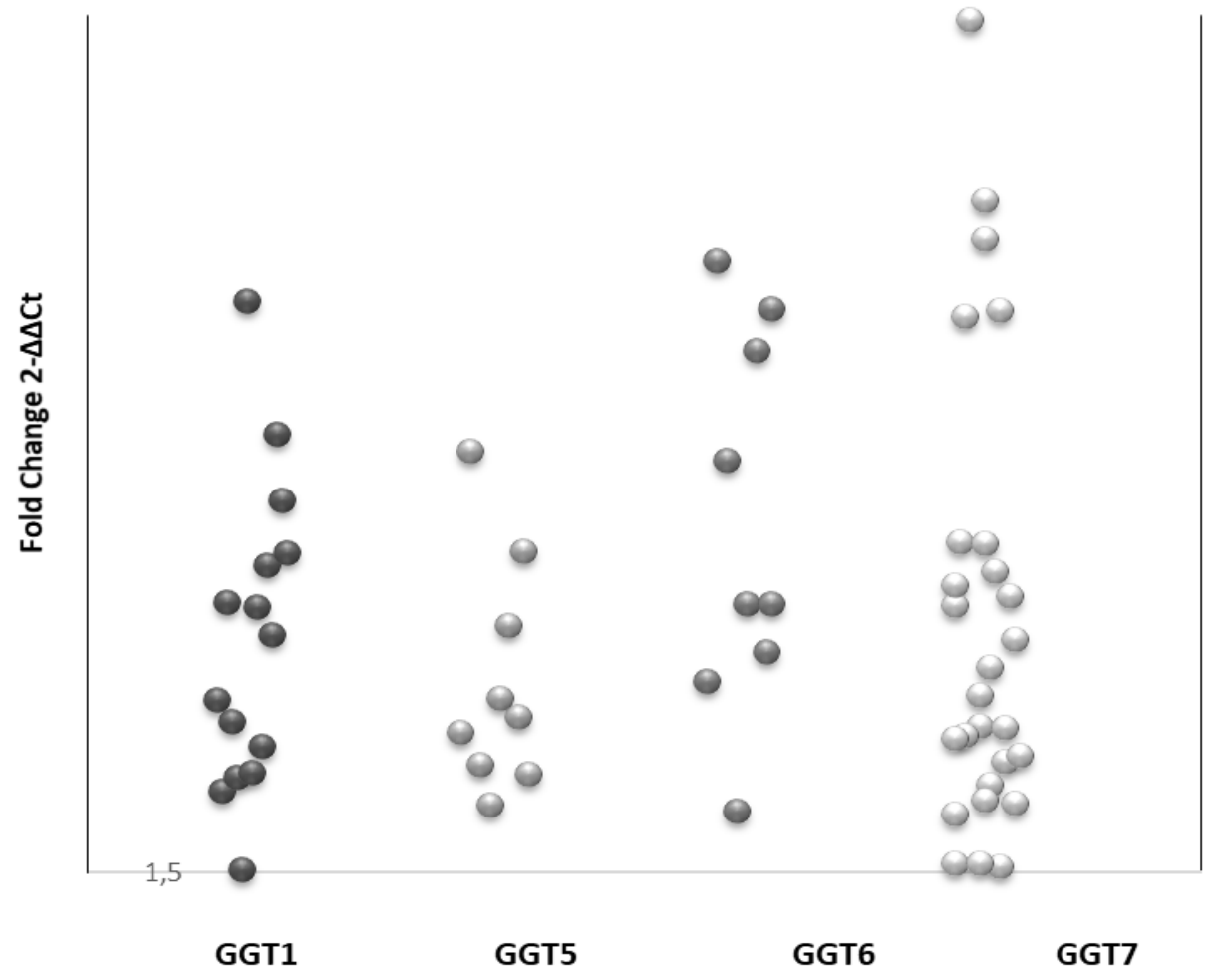

The $\mathrm{Y}$-axis indicates the fold change in gene expression, and the $\mathrm{X}$-axis represents the patients. a 1.5 fold increase in gene expression in tumor tissue was considered as overexpression.

Figure 2. Scatter Plot of Expressions of GGT1 (a), GGT5 (b), GGT6 (c), and GGT7 (d) in Molecular Subgroups of Breast Cancer

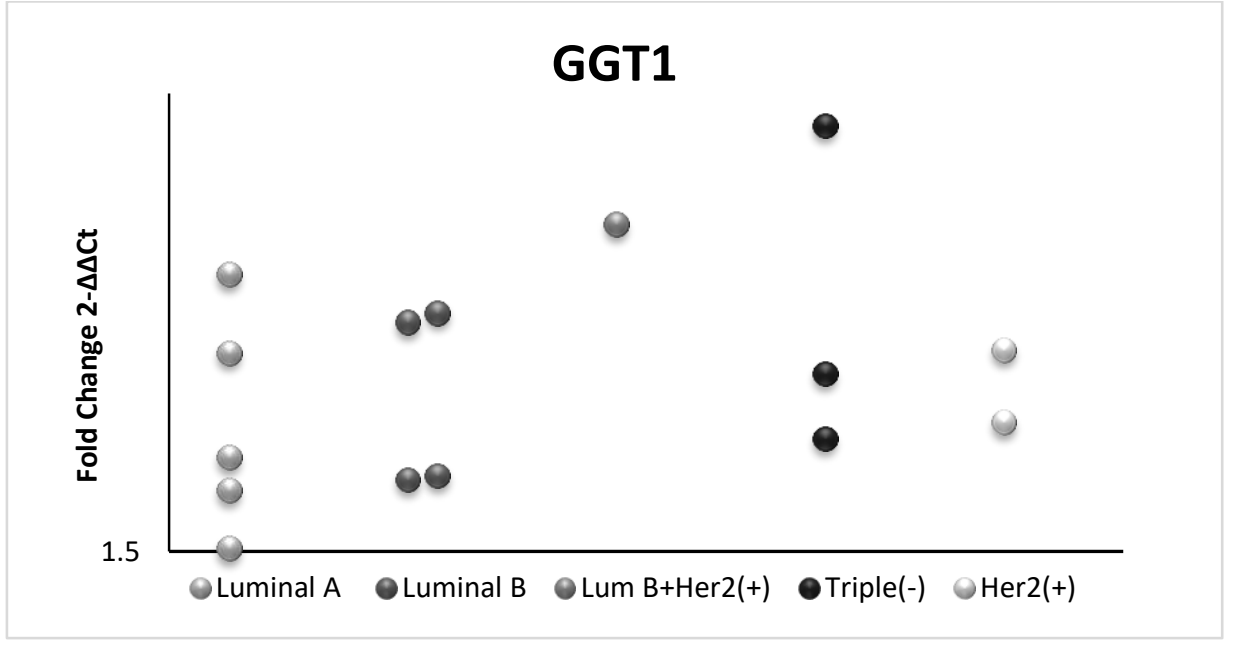

a 


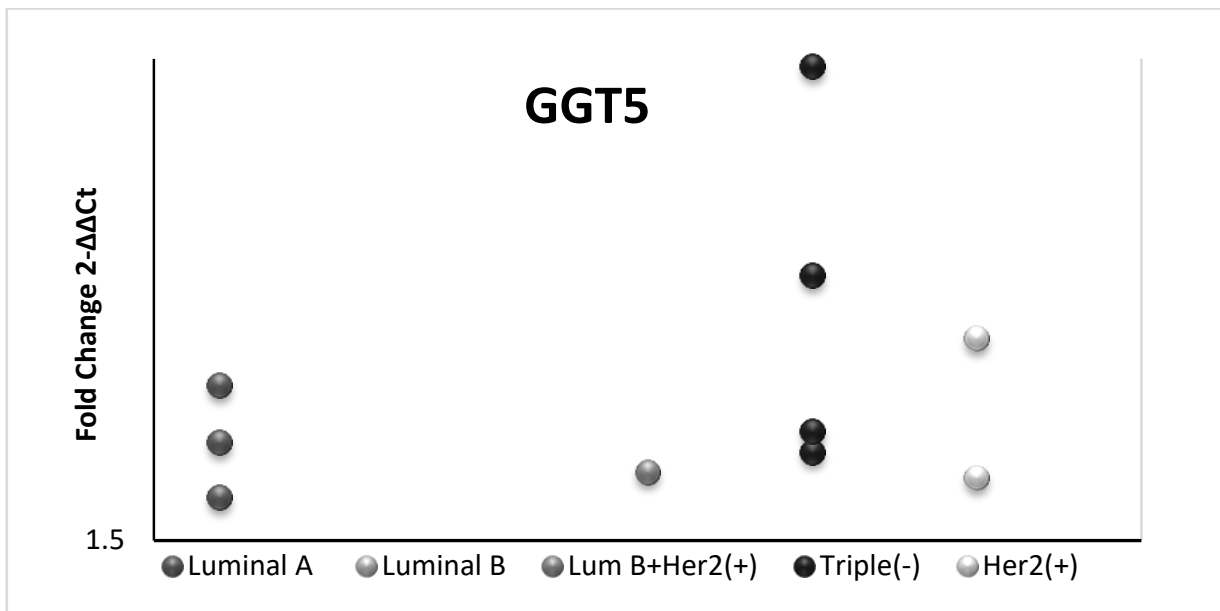

$\mathrm{b}$

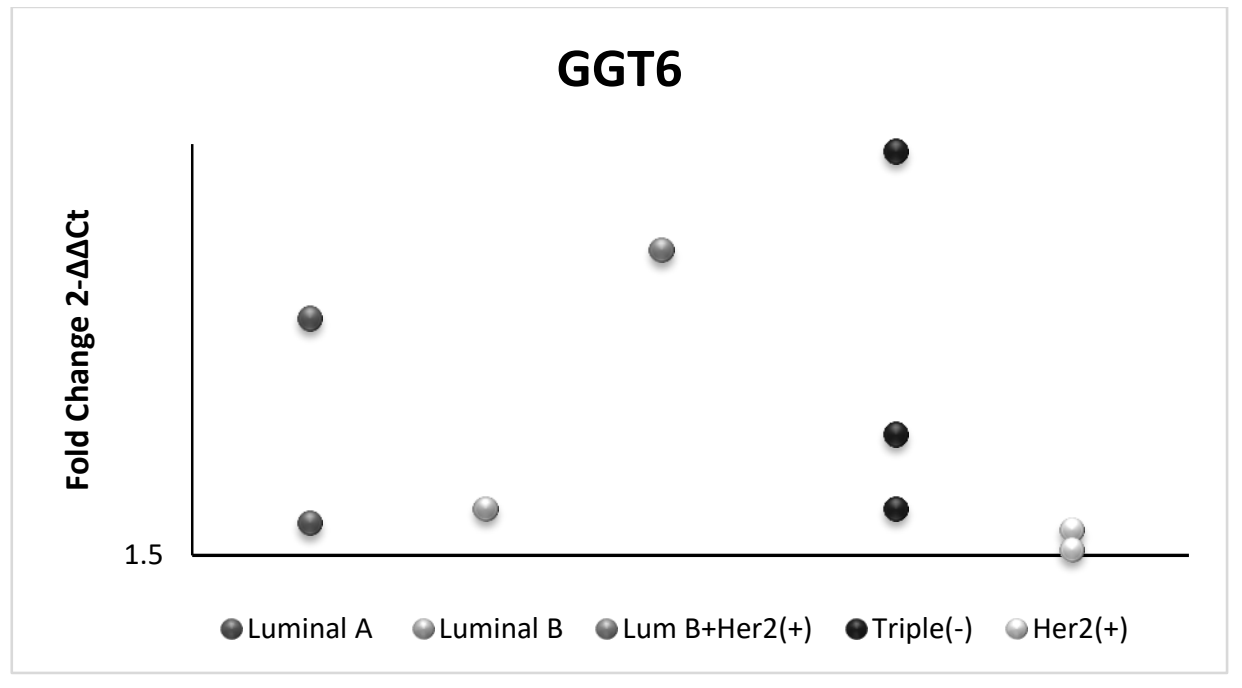

c

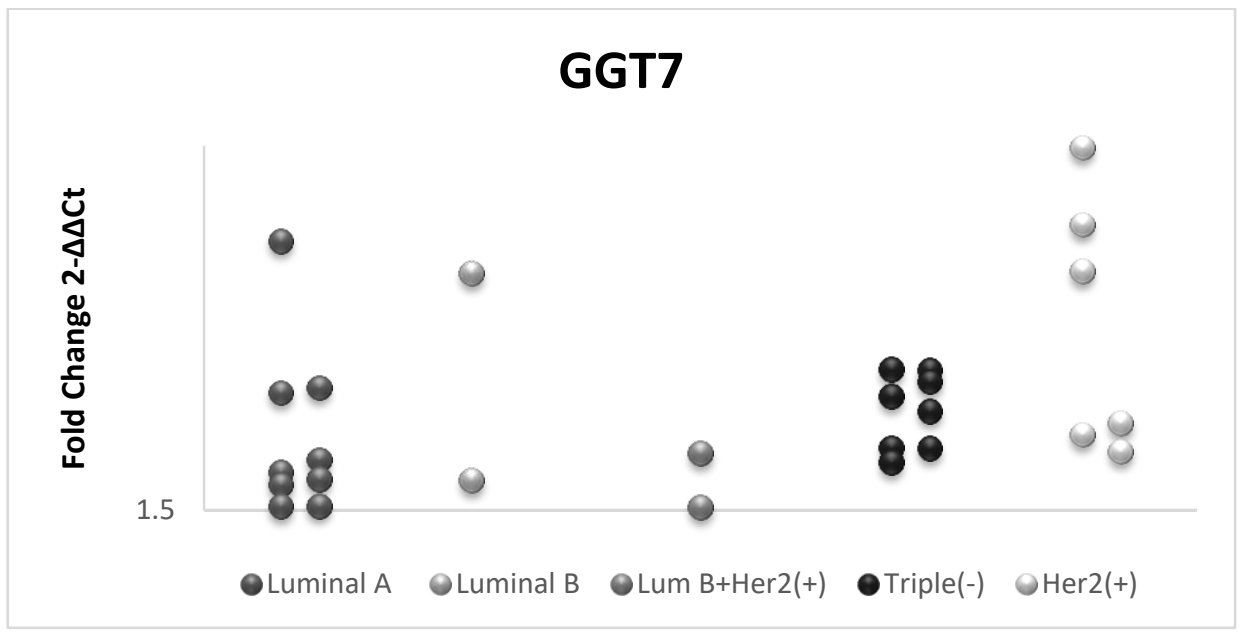

d

The $\mathrm{Y}$-axis indicates the fold change in gene expression, and the $\mathrm{X}$-axis represents the patients. $\mathrm{A}$ 1.5 fold increase in gene expression in tumor tissue was considered as overexpression. 
Table 3. Comparison of Median ( \pm Standard Deviation, SD) GGT5 and GGT7 Expressions in Normal and Tumor Tissues in Breast Cancer (Wilcoxon Rank-Sum Test, $P$-Value <0.05)

\begin{tabular}{|l|c|c|c|}
\hline & \multicolumn{2}{|c|}{ mRNA Expression Levels $(\Delta \Delta \mathrm{Cq})$, Median $(\mathrm{SD})$} & \\
\hline & Matched-Normal Tissue & Tumor Tissue & $\mathrm{P}$ \\
\hline GGT1 & $0.1414(0.2500)$ & $0.2048(0.9422)$ & 0.020 \\
\hline GGT5 & $0.0187(0.0390)$ & $0.0328(0.1350)$ & 0.145 \\
\hline GGT6 & $0.1942(0.3287)$ & $0.1366(0.3619)$ & 0.045 \\
\hline GGT7 & $0.1349(0.1872)$ & $0.2132(0.2500)$ & 0.003 \\
\hline
\end{tabular}

\section{Discussion}

In this study, we examined the expression of GGT genes in the tissues of breast cancer patients and evaluated their potential as biomarkers in detection strategies. For this purpose, we compared mRNA expression in normal and tumor tissues of the same patients.

GGT1, which has a precursor peptide sequence containing 569 amino acids, is an enzyme with important functions in extracellular glutathione metabolism, LTC4 catabolism, and glutathione homeostasis. GGT1 is the most expressed in the normal tissues of kidney, duodenum, small intestine, and prostate (Heisterkamp et al. 2008). In this study it was observed that GGT1 was significantly expressed in breast cancer when compared with the control. The expression of tumor tissue GGT1 has also been shown in various cancers such as prostate (Kawakami et al. 2017), epithelial (Lukic et al. 2016), lung (Hino et al. 2016), colorectal (Palaniappan et al. 2016), overian (Mahata 2006), liver (Pavesi et al. 1989), and breast (Banneau et al. 2010).

GGT5 is a protein that is clearly homologous to GGT1 at the amino acid level, with significant deletion due to nucleotide identity. GGT5 also had a protein with enzymatic activity (Heisterkamp et al. 2008). Previous studies using GGT1 and GGT5 null mutants showed that GGT1 is the main enzyme involved in glutathione metabolism (Carter et al. 1997, 1998), whereas GGT5 is mainly involved in LTC4 metabolism (Han et al. 2002). Studies in cDNA libraries have shown that the mRNA of GGT5 is extensively expressed in normal tissues such as adrenal glands, adipose tissue, lymph nodes, kidneys, and bile ducts (NCBI 2019a). In addition, increased expression of GGT5 has also been observed in kidney, glioma, and esophageal cancers (Heisterkamp et al. 2008). GGT6 was identified in rats by Puente and Lopez-Otin and was included in the threonine protease family (2004). The function of GGT6 as an enzyme has not yet been described and there are no studies showing its translation into a potential protein (Heisterkamp et al. 2008). In normal tissues, GGT6 expression is most abundant in the colon, kidney, duodenum, and small intestine. On the other hand, expression of GGT6 has been observed to increase in the adrenal, colorectal, and breast cancers (NCBI 2019b).

GGT7 is the nucleotide sequence encoding the peptide containing 662 amino acids (Kyoto Encyclopedia of Genes and Genomes 2019, NCBI 2018a). Although GGT7 shows significant similarity to GGT1 in terms of amino acid sequencing, 
there is a significant lack of nucleotide identity, and GGT1 and GGT7 are therefore included in separate families (Heisterkamp et al. 2008). Its functions have not yet been fully elucidated and are thought to be involved in enzymatic activities regulated by leukotriene synthesis, glutathione metabolism, or glutamyl transfer. GGT7 is extensively expressed in normal tissues such as brain, thyroid, ovarian, and prostate (NCBI 2018b). However, expression of GGT7 has been observed to increase in bladder, glioma, head and neck, lung, and PNET cancers (Bui et al. 2015).

There is only one previous publication from our group, showing the expressions of GGT1, GGT5, and GGT6 mRNA in a small group of different types of cancer, including breast cancer (Yardım-Akaydın et al. 2017).

This study was designed to examine these three GGT genes, by adding GGT7, in only breast cancer patients according to molecular subtypes. Two reports have been published focusing on GGT1 expression in molecular apocrine breast cancer (MABC) subtype of breast cancer (Banneau et al. 2010, Guo et al. 2015). Furthermore, it was observed that most patients in MABC subtype, which included tumors with ER-/PR-/HER2- (triple-negative breast cancer, TNBC) and ER-/PR-/HER2+ (HER2-overexpression) (Liu et al. 2016), showed GGT1 expression, while its expression was observed in only $1.5-9.6 \%$ of non-MABC cases. Except ours, there is no published clinical study showing GGT5 and GGT6 expressions in breast cancer or any other cancer. However, in a study examining the expression of GGT7 in glioblastoma, which is an aggressive malignant tumor, it was determined that patients with high GGT7 expression had a better prognosis than patients with low expression (Bui et al. 2015). It is also reported that, GGT7 played a role in tumorigenesis with an anti-oxidative regulating effect and GGT7 reduction has been shown to increase the amount of cellular reactive oxygen species and thus induce tumor formation and growth (Bui et al. 2015). According to the results of our study, GGT1, GGT5, and GGT7 expressions increased in tumor tissue compared to matched-normal tissues, whereas GGT6 decreased. On the other hand, GGT7 overexpression was observed in most of the patients in all molecular subgroups, except for Luminal B groups.

\section{Conclusion}

GGT is an enzyme that plays important roles in both glutathione metabolism, which is associated with oxidative stress and drug resistance, and leukotriene synthesis. Therefore, it is important to determine the type of GGT responsible for the increase in GGT activity, which is considered a risk factor in breast cancer. This study showed that there were GGT1, GGT5, GGT6, and GGT7 mRNA expressions in molecular subtypes of breast cancer. According to the results, it is suggested that GGT7 may have marker potency in breast cancer, especially in the luminal A, HER2 positive, and triple negative groups. In our research laboratory, we continue our studies to examine potential functions of GGT7 gene in breast cancer. 


\section{Acknowledgments}

This study was supported by Gazi University Research Fund (Project No.: 02/2017-02).

\section{References}

Banerji S, Cibulskis K, Rangel-Escareno C, Brown KK, Carter SL, Frederick A et al. (2012) Sequence analysis of mutations and translocations across breast cancer subtypes. Nature 486(Jun): 405-409.

Banneau G, Guedj M, MacGrogan G, de Mascarel I, Velasco V, Schiappa R et al. (2010) Molecular apocrine differentiation is a common feature of breast cancer in patients with germline PTEN mutations. Breast Cancer Research 12(4): R63.

Brenda (2019) The comprehensive enzyme information system. Retrieved from: https:// www.brenda-enzymes.org/enzyme.php?ecno=2.3.2.2.

Bui TT, Nitta RT, Kahn SA, Razavi SM, Agarwal M, Aujla P et al. (2015) GammaGlutamyl transferase 7 is a novel regulator of glioblastoma growth. BMC Cancer 15(Apr): 225.

Carey LA, Perou CM, Livasy CA, Dressler LG, Cowan D, Conway K et al. (2006) Race, breast cancer subtypes, and survival in the Carolina Breast Cancer Study. JAMA 295(21): 2492-2502.

Carter BZ, Wiseman AL, Orkiszewski R, Ballard KD, Ou CN, Lieberman MW (1997) Metabolism of leukotriene C4 in gamma-glutamyl transpeptidase-deficient mice. Journal of Biological Chemistry 272(19): 12305-12310.

Carter BZ, Shi ZZ, Barrios R, Lieberman MW (1998) Gamma-glutamyl leukotrienase, a gamma-glutamyl transpeptidase gene family member, is expressed primarily in spleen. Journal of Biological Chemistry 273(43): 28277-28285.

Cheang MC, Chia SK, Voduc D, Gao D, Leung S, Snider J et al. (2009) Ki67 index, HER2 status, and prognosis of patients with luminal B breast cancer. Journal of the National Cancer Institute 101(10): 736-750.

Dent R, Trudeau M, Pritchard KI, Hanna WM, Kahn HK, Sawka CA et al. (2007) Triplenegative breast cancer: clinical features and patterns of recurrence. Clinical Cancer Research 13(15): 4429-4434.

Fentiman IS, Allen DS (2010) Gamma-glutamyl transferase and breast cancer risk. Breast Cancer Research 103(1): 90-93.

Fornaciari I, Fierabracci V, Corti A, Aziz Elawadi H, Lorenzini E, Emdin M et al. (2014) Gamma-glutamyltransferase fractions in human plasma and bile: characteristic and biogenesis. Plos One 9(2): e88532.

Guo W, Wang W, Zhu Y, Zhu X, Shi Z, Wang Y (2015) HER2 status in molecular apocrine breast cancer: associations with clinical, pathological, and molecular features. International Journal of Clinical and Experimental Pathology 8(7): 8008-8017.

Han B, Luo G, Shi ZZ, Barrios R, Atwood D, Liu W et al. (2002) Gamma-glutamyl leukotrienase, a novel endothelial membrane protein, is specifically responsible for leukotriene D(4) formation in vivo. American Journal of Pathology 161(2): 481-490.

Hanigan MH (2014) Gamma-glutamyl transpeptidase: redox regulation and drug resistance. Advances in Cancer Research 122(Jun): 103-141.

Heisterkamp N, Groffen J, Warburton D, Sneddon TP (2008) The human gammaglutamyltransferase gene family. Human Genetics 123(4): 321-332. 
Hino H, Kamiya M, Kitano K, Mizuno K, Tanaka S, Nishiyama N et al. (2016) Rapid cancer fluorescence 1maging using a gamma-glutamyltranspeptidase-specific probe for primary lung cancer. Translational Oncology 9 (3): 203-210.

InterPro (2017) Gamma-glutamyltranspeptidase. Retrieved from: http://www.ebi.ac.uk/ interpro/entry/InterPro/IPR000101/?q=ggt\%2C\%3A\%20Accessed\%202017.

Kawakami K, Fujita Y, Matsuda Y, Arai T, Horie K, Kameyama K et al. (2017) Gammaglutamyltransferase activity in exosomes as a potential marker for prostate cancer. BMC Cancer 17(1): 316.

Kyoto Encyclopedia of Genes and Genomes. 2019. Retrieved from https://www.genome. jp/dbget-bin/www_bget?pps:100979819

Liu XZ, Yang Y, Feng XL, Shen HH, Liu J, Liu X et al. (2016) Early versus late distant metastasis and adjuvant chemotherapy alone versus both radiotherapy and chemotherapy in molecular apocrine breast cancer. Oncotarget 7(31): 48905-48917.

Lukic A, Ji J, Idborg H, Samuelsson B, Palmberg L, Gabrielsson S (2016) Pulmonary epithelial cancer cells and their exosomes metabolize myeloid cell-derived leukotriene C4 to leukotriene D4. Journal of Lipid Research 57(9): 1659-1669.

Mahata P (2006) Biomarkers for epithelial ovarian cancers. Genome Informatics 17(2): 184-193.

MyBioSource (2006) GGT1 protein: gamma-glutamyl transferase protein. Retrieved from: https://www.mybiosource.com/ggt1-active-protein/gamma-glutamyl-transferase/730 93.

NCBI (2018a) Gamma-glutamyltransferase 7 [Homo sapiens]. Retrieved from: https:// www.ncbi.nlm.nih.gov/protein/AAI41844.1.

NCBI (2018b) GGT7 gamma-glutamyltransferase 7 [Homo sapiens (human)]. Retrieved from: https://bit.ly/3hsmpTF.

NCBI (2019a) GGT6 gamma-glutamyltransferase 6 [Homo sapiens (human)]. Retrieved from: https://www.ncbi.nlm.nih.gov/gene/124975.

NCBI (2019b) GGT5 gamma-glutamyltransferase 5 [Homo sapiens (human)]. Retrieved from: https://bit.ly/2UIKv2P.

Palaniappan A, Ramar K, Ramalingam S (2016) Computational identification of novel stage-specific biomarkers in colorectal cancer progression. Plos One 11(5): 0156665

Pavesi F, Lotzniker M, Scarabelli M, Garbagnoli P, Moratti R (1989) Efficiency of composite laboratory tests in the diagnosis of liver malignancies. International Journal of Bio Marker 4(3): 163-169.

Perou CM, Sorlie T, Eisen MB, van de Rijn M, Jeffrey SS, Rees CA et al. (2000) Molecular portraits of human breast tumours. Nature 406(6797): 747-752.

Pfaffl MW (2001) A new mathematical model for relative quantification in real-time RTPCR. Nucleic Acids Research 29(9): e45.

Puente XS, Lopez-Otin CA (2004) Genomic analysis of rat proteases and protease inhibitors. Genome Research 14(4): 609-622.

Rouzier R, Perou CM, Symmans WF, Ibrahim N, Cristofanilli M, Anderson K et al. (2005) Breast cancer molecular subtypes respond differently to preoperative chemotherapy. Clinical Cancer Research 11(16): 5678-5685.

Shackshaft L, Van Hemelrijck M, Garmo H, Malmstrom H, Lambe M, Hammar N et al. (2017) Circulating gamma-glutamyl transferase and development of specific breast cancer subtypes: findings from the Apolipoprotein Mortality Risk (AMORIS) cohort. Breast Cancer Research 19(22): 2-5.

Staudigl C, Concin N, Grimm C, Pfeiler G, Nehoda R, Singer CF et al. (2015) Prognostic relevance of pretherapeutic gamma-glutamyltransferase in patients with primary metastatic breast cancer. Plos One 10(4): e0125317. 
Tran B, Bedard PL (2011) Luminal-B breast cancer and novel therapeutic targets. Breast Cancer Research 13(6): 221.

Türkyilmaz M, Hacikamiloğlu E, Baran Deniz E, Boztaş G, Dündar S, Kavak Ergün A et al. (2018) Türkiye Kanser İstatistikleri 2015 (Turkey Cancer Statistics 2015). Ankara: Halk Sağlığı Genel Müdürlüğ̈̈, Sağlık Bakanlığı.

Van Hemelrijck M, Jassem W, Walldius G, Fentiman IS, Hammar N, Lambe M et al. (2011) Gamma-glutamyltransferase and risk of cancer in a cohort of 545,460 persons - the Swedish AMORIS study. European Journal of Cancer 47(13): 2033-2041.

Yang XR, Sherman ME, Rimm DL, Lissowska J, Brinton LA, Peplonska B et al. (2007) Differences in risk factors for breast cancer molecular subtypes in a population-based study. Cancer Epidemiology, Biomarkers and Prevention 16(3): 439-443.

Yardım-Akaydın S, Deviren C, Miser-Salihoğlu E, Calışkan-Can E, Atalay MC (2017) mRNA expressions of gamma-glutamyl transferase genes in different types of cancer. Fabad Journal of Pharmaceutical Sciences 42(1): 21-28. 
